\title{
Effect of Self-Assembled Monolayers on the Performance of Organic Photovoltaic Cells
}

\author{
Hanène Bedis \\ ${ }^{1}$ UMAO, Faculté des Sciences de Tunis, Campus Universitaire, Tunis, Tunisia; ${ }^{2}$ ITODYS, 15 Rue Jean-Antoine de Baïf , Paris, \\ France. \\ Email: hanene.bedis@fst.rnu.tn
}

Received April $1^{\text {st }}, 2011$; revised May $5^{\text {th }}, 2011$; accepted May $14^{\text {th }}, 2011$.

\begin{abstract}
The improvement of the performance of organic photovoltaic cells (OPVCs) and the photogeneration process in these devices may occur via multiple mechanisms depending on their structure and/or architecture. For this purpose we investigate how self-assembled monolayers of thiol molecules $\left(\mathrm{C}_{12} \mathrm{H}_{25} \mathrm{SH}\right.$ and $\left.3 \mathrm{~T}\left(\mathrm{CH}_{2}\right)_{6} \mathrm{SH}\right)$ and benzoic acid molecules (ABA and NBA) affect the efficiency and the photogeneration of free carriers in a sexithiophene based photovoltaic cells. Firstly, we provide the results of absorption spectra for samples with SAM of thiol that show there effect on orientation of $6 T$ molecules on these structures and the organization degree of the thiol molecules on ITO substrate. Afterward, we describe from current vs. applied voltage after illumination, the enhancement of the performance of these cells. In the second, we study the effect of SAM of benzoic acids molecules on the photovoltaic behavior. A theoretical model is used for quantitative description of the open circuit voltage as a function of carrier's generation rates at the electrodes. The results of $I-V$ characterization under illumination show that open circuit voltage as well as short circuit current is dramatically affected by the dipolar layer. The orientation and the magnitude of dipole moment of benzoic acid molecules are the crucial factors that affect the organic photovoltaic parameters.
\end{abstract}

Keywords: SAMs, Oligothiophene, Photovoltaïc Cell, Photogeneration, Efficiency, Performance

\section{Introduction}

Organic semiconductors materials are potential candidates in electronics industry, more diversified and less expensive. Indeed, the fact that is a synthetic materials contrary to the mineral semiconductors, allows to modeling the constitutive molecules to adjust a very precise property. It is by proceeding that we were able to conceive a multitude of electronic structures such as organic light-emitting diodes (OLEDs) which cover the totality of the visible spectral field and organic photovoltaic cells (OPVCs).

Organic photovoltaic cells are gaining considerable interest motivated by a steady enhance of their conversion efficiency during the last decade [1-3]. Nevertheless, their operating mechanisms are still subject of controversial theories. In fact, the observed photocurrent in OPVCs is an experimental evidence of free photocarrier production. Nonetheless, the origin of photogenerated carriers that are responsible of the observed photocurrent may be attributed to mainly three mechanisms. The first is the ionization of tight bound excitons or the Frenkeltype excitons in the bulk which is described by Onsager theory $[4,5]$. In this mechanism Frenkel excitons lead to the formation of charge transfer excitons resulting in geminate pairs of photogenerated carriers lying on adjacent molecules. The geminate pairs escape the strong columbic attraction under the effect of built-in electric field $\mathrm{E}$ at a given temperature $\mathrm{T}$ with the probability $\mathrm{P}(\mathrm{E}, \mathrm{T})$. The second mechanism occurs at the interface with the electrodes. In this mechanism one of the geminate pair carriers passes through the electrode and the other is left "free" to contribute to photocurrent. The last mechanism is attributed to detrapping of trapped carriers. In this mechanism photogenerated tight bound excitons transfer enough energy to trapped charges by colliding them. Actually, all of the three described mechanisms may occur simultaneously but in some cases one among them may be preponderant on the two others as for example the case of materials with low mobility, in which bulk photogeneration may be ignored because their short lifetime and photogenerated carriers in the bulk that are 
far from the electrodes disappear rapidly and minimizing the photocurrent.

The development of organic photovoltaic cells (OPVCs) is still a matter of research despite their low efficiency relatively to mineral ones which is precisely a crucial factor for their commercialization. This is due to mainly two reasons. The first one may be qualified as historical and is related to the youthfulness of organic optoelectronics by comparison to minerals. The second one is physical and is due to mainly two reasons:

- The first one is the low dielectric constant of organic semiconductors $(\sim 3)$ relatively to that of inorganic ones $(\sim 10)$. This property makes photo-excited electron-hole geminate pairs much more bound, due to columbic interaction, in organic materials indeed excitons in organic semiconductors are Frenkel-type with a binding energy in the order of some tenths of $\mathrm{eV}$ [5].

- The second one is the low mobility of carriers in organic semiconductors which inhibits short lifetime carriers from attaining elec trodes [6].

The relatively high binding energy prevents these photogenerated species from dissociating which reveals low free carrier production efficiency. The most probable sketch to account for photocurrent observed experimenttally in organic solar cells is that free carriers are produced from dissociation of those tightly bound geminate pairs by interaction with the metal/Semiconductor interface [5].

Self Assembled Monolayers (SAMs) which were widely used in OLEDs are supposed to induce modification of metallic electrode work function leading to significant improve of carrier injection into the organic semiconductor. Another advantage in using SAM layers at electrode interfaces is to improve the adhesion of the organic film onto the metal or the oxide electrode [7]. Then, the quality of the interface reflected by the degree of the structural order, has been improved substantially by using self-assembled monolayer (SAM) at the interface either in the organic transistor [8], the organic diode [9] or the organic light emitting diode [10].

We are interested in this work on photovoltaic cells based of the $\alpha$-sexithiophene (6T) molecules (Figure 1(a)). The characteristics of these molecules apart their stability and facility of synthesis, they are essentially motivated by their structural order, the degree of organization and the big purity which offers to oligomeres contrary to polymers. These molecules present by their simplicity a better understanding of the optoelectronics phenomena and allow to modeling the behavior of polythiophenes.

We present in this article the methods for improving performance of sexithiophene based photovoltaic cell

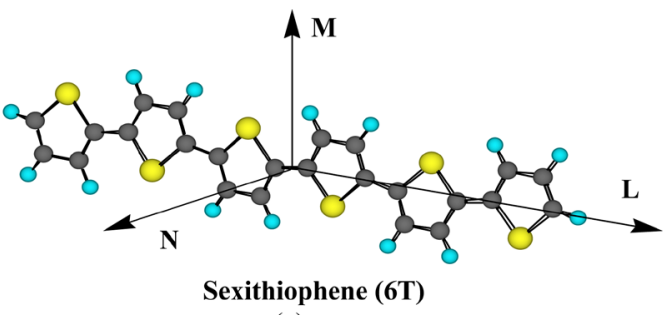

(a)

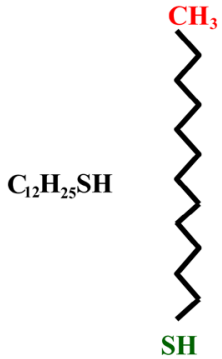

(b)

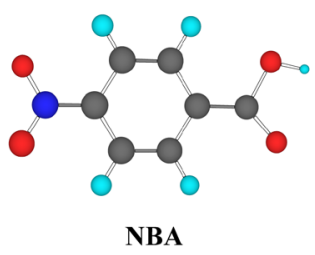

(d)

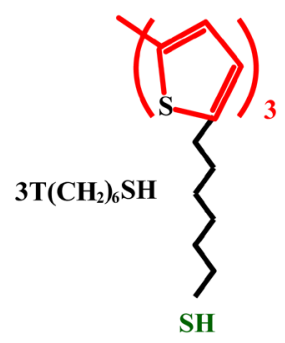

(c)

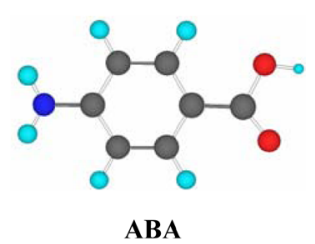

(e)
Figure 1. Chemical structure of: (a) sexithiophene, (b) Dodecanthiol $\left(\mathrm{C}_{12} \mathrm{H}_{25} \mathrm{SH}\right)$, (c) Thiol with head group the terthiophene (3T $\left.\left(\mathrm{CH}_{2}\right)_{6} \mathrm{SH} / 6 \mathrm{~T}\right)$, (d) p-Nitro-benzoic acid NBA and (e) p-Amino-benzoic acid ABA.

through introduce a self-assembled monolayer of thiols molecules differed with functional groups $\left(\mathrm{C}_{12} \mathrm{H}_{25} \mathrm{SH}\right.$ and $3 \mathrm{~T}\left(\mathrm{CH}_{2}\right)_{6} \mathrm{SH}$ ) (Figure 1(b) and Figure 1(c)) and benzoic acid molecules (Amino-Benzoic Acid and Nitro-Benzoic Acid) (Figure 1(d) and Figure 1(e)) on ITO in order to control photocarrier generation at the interface ITO/organic and to provide an increase in device efficiency. In this study, the evaporate 6T both on SAMs devices were characterized electrically after illumination. We describe from current vs. applied voltage, the enhancement of devices efficiencies and we can estimate their photovoltaic parameters. A theoretical model is used for quantitative description of the open circuit voltage as a function of carrier's generation at the electrodes, and can explain the effect of orientation and the magnitude of dipole moment of SAM of benzoic acid on the photogeneration rate of free carriers and their effect on the organic photovoltaic parameters.

\section{Experimental}

\subsection{Preparation of ITO}

The purpose of the preparation of the ITO is to eliminate 
the impurities and to increase its surface reactivity and the interactions with the grafted molecules. For the whole of our experimental work, the glassmaking and the whole set of our manipulating tools are carefully cleaned in order to avoid to the maximum any contamination which may trouble grafting of SAM.

Prior to SAMs grafting ITO substrates, provided by SOLEMS, were carefully cleaned according to the following protocol: first the samples are immerged in an ultrasound bath of ultrapure water for $30 \mathrm{mn}$, second they are rinsed in pure $\mathrm{NaOH}(30 \%)$ during $15 \mathrm{mn}$ and third, they are immerged in pure sulphuric acid (98\%) during $1 \mathrm{mn}$. After that the samples are rinsed again in an ultrasound bath of ultra pure water during $1 \mathrm{mn}$. This chemical treatment is used in order to render ITO surface more hydrophilic and reactive with respect to grafted SAM.

\subsection{Preparation of SAM and Devices Structure}

SAMs are obtained by solution dipping of cleaned ITO substrates according to the following procedure: we prepared two solutions with the molecule shown in Figure 1.

To deposit SAMs on the cleaned ITO substrates, we used in the first for thiol molecules a pure solution of $2 \mathrm{ml}$ of Dodecanethiol: $\mathrm{C}_{12} \mathrm{H}_{25} \mathrm{SH}$ (Figure 1(a)), and a prepared solution of $3 \mathrm{~T}\left(\mathrm{CH}_{2}\right)_{6} \mathrm{SH}$ (Figure 1(b)) in benzene with a concentration of $10^{-3} \mathrm{~mol} / \mathrm{l}$. The samples of cleaned ITO substrates were left for one week in these solutions in order to maximize the SAM graftin g [11]. Afterward the samples were rinsed in pure ethanol $\left(\mathrm{C}_{2} \mathrm{H}_{6} \mathrm{O}\right.$ (96\%)) in an ultrasonic bath, then dried with argon. All the experimental steps were carried out at room temperature. For acid molecules, we used pure solution of $2 \mathrm{ml}$ of 4-Aminobenzoic acid (ABA), 4-Nitrobenzoic acid (NBA) Figure 1(d) and Figure 1(e). All the experimental steps were performed at room temperature.

In order to obtain comparable results either between SAM coated ITO and bare ITO we performed both sexi- thiophene (6T) and aluminum (Al) coatings in the same conditions. We deposited $80 \mathrm{~nm}$ of $6 \mathrm{~T}$ on five substrates: four of them were coated with SAMs (thiols and benzoic acids) the last one is just for bare cleaned ITO. A semi-transparent $20 \mathrm{~nm}$ thick aluminum cathode was finally deposited through a shadow mask under $10^{-6}$ torr leading to final structures ITO/SAM/6T/Al (Figure 2) and ITO/6T/Al. Each sample consisted of two pixels each of which had a rectangular shape of $2 \mathrm{~mm} / 4 \mathrm{~mm}$.

\subsection{Electrical and Optical Characterization}

Electrical measurements were performed with a Keithley 4200-SCS Semiconductor Characterization System. For I-V measurement under illumination, the first irradiating light source was a $150 \mathrm{~W}$ Tungsten lamp for the first series of samples with SAM of thiols and the second irradiating light source was a $150 \mathrm{~W}$ Xenon arc lamp (model $150 \mathrm{~W} / 1 \mathrm{XBO}$, Osram).

Absorption spectra have been recorded with a Carry 500 UV-VIS-NIR spectrophotometer that directly gives the variation of optical density D.O or absorbance vs. wave length of thin film of 6T. All measurements are taken in the ambient air and at room temperature on the optical seat by illumination of the cell through the semi-transparent Aluminium electrode.

\section{Theory}

In this section we calculate the open voltage for the sample geometry described above. We assume that for applied voltages $V=V_{o c}$ the charge density inside the sample is low enough that it does not cause any band bending, it remains localized at the contact, the photocurrent is equal to zero and the electric field remains uniform and equal to $\left(V_{b i}-V\right) / d$, where $d$ is the thickness of the poly- mer layer.

The carrier conduction of in this case is limited of hole and current densities $J_{h}$ of hole respectively is:

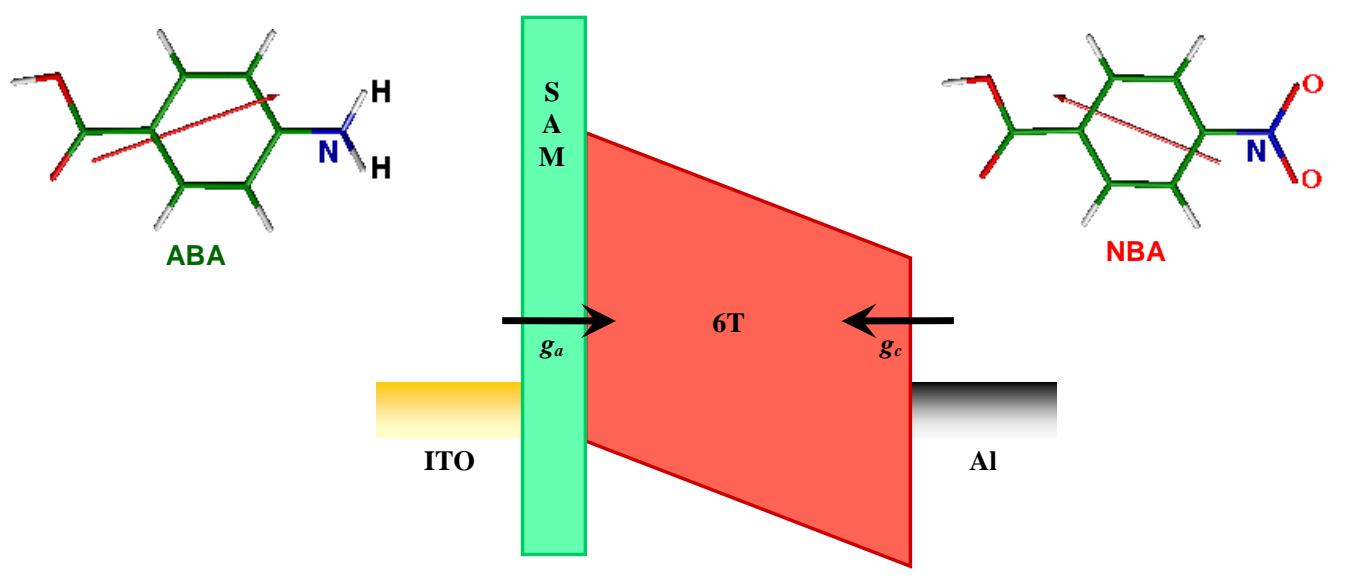

Figure 2. Energy diagram of ITO/SAM/6T/Al structure. 


$$
J=-e \mu_{h} p(x) \frac{V_{b i}-V}{d}+e D_{h} \frac{\partial p(x)}{\partial x}
$$

where $e$ is the elementary charge, $\mu_{h}$ is the hole mobility, $D$ is the diffusion coefficient and $p$ is the hole density. Solving the Equation (1) we get the hole density [12]:

$$
p(x)=\frac{p_{c}-p_{a}}{e^{-q d}-1} e^{-q x}+\frac{p_{a} e^{-q d}-p_{c}}{e^{-q d}-1}
$$

where the subscripts " $a$ " and " $c$ " denote charge concentrations at the anode $(x=0)$ and the cathode $(x=d)$, respectively, and $q=\frac{1}{d} \frac{e\left(V_{b i}-V\right)}{k T}$.

Using the Einstein relation between the mobility and the diffusion coefficient one get the dark current:

$$
J_{D}=e\left(\frac{V_{b i}-V}{d}\right) \frac{\left(\mu p_{a}-\mu p e^{q d}\right)}{e^{q d}-1}
$$

The total current density under illumination is in the following form:

$$
J_{t}=J_{D}+J_{L}
$$

where the steady state current density in the dark is:

$$
J_{D}=e\left(\frac{V_{b i}-V}{d}\right) \frac{\left(\mu p_{a}-\mu p_{c} \mathrm{e}^{q d}\right)}{e^{q d}-1}
$$

Since exctions in organic materials are tightly bound, the basic charge generation mechanism for photoconductivity is believed to involve dissociation of the excited state via transfer of charge to the metal electrode, leaving the other charge free inside the organic layer [12]. Thus, in order to calculate the current density under illuminetion one can neglect bulk photogeneration and assume that only photogenerated carriers at the electrodes contribute to photocurrent. Therefore, $J_{L}$ is obtained by substituting respectively $p_{a}$ and $p_{c}$ in $J_{D}$ by $g_{a} I$ and $g_{c} I$, where I stand for the illumination intensity and $g_{a}$ and $g_{c}$ are the density of photogenerated holes respectively at the anode and the cathode. Thus $\mathrm{J}_{\mathrm{L}}$ is written as:

$$
J_{L}=e\left(\frac{V_{b i}-V}{d}\right) \frac{\left(\mu g I-\mu g I e^{q d}\right)}{e^{q d}-1}
$$

Then, the total current density is written in the form:

$$
J_{t}=e \mu_{h}\left(\frac{V_{b i}-V}{d}\right)\left[\frac{p_{a}+g_{a} I-\left(p_{c}+g_{c} I\right) e^{q d}}{e^{q d}-1}\right]
$$

Or at $V=V_{o c}$, the total current density equals zero then we get the relation between the built-in potential and the open circuit voltage as follows:

$$
V_{o c}=V_{b i}-\frac{k T}{e} \ln \left(\frac{p_{a}+g_{a} I}{p_{c}+g_{c} I}\right)
$$

Since generally organic semiconductors are known to be excellent photoconductors that is photocurrent is orders of magnitude greater that dark current, then one may neglect $p_{a}$ and $p_{c}$ with respect to $g_{a} I$ and $g_{c} I$ respectively leading to the expression:

$$
V_{o c}=V_{b i}-\frac{k T}{e} \ln \left(\frac{g_{a}}{g_{c}}\right)
$$

On the other hand, the built-in potential $V_{b i}$ in conjugated non-doped thin film devices is proportional to the workfunction difference between the cathode and the anode.

$$
V_{b i}=\frac{1}{e}\left(\phi_{\mathrm{ITO}}-\phi_{\mathrm{Al}}\right)
$$

where $\phi_{\text {Iто }}$ and $\phi_{\mathrm{Al}}$ are the workfunctions of the anode (ITO) and of the cathode (Al). In the case of SAM coated electrodes one should take into account the workfunction shift due to dipole layer introduced by dipolar character of SAM molecules. Then the Potential energy shift owing to a dipolar SAM layer at the ITO surface is given by [13]:

$$
\Delta \phi=\frac{e \Gamma \mu_{D}}{\varepsilon_{0} \varepsilon}
$$

where $\Gamma$ is the number of molecules by unit surface $(2 \times$ $10^{18} \mathrm{~m}^{-2}$ for ABA molecule and $1.3 \times 10^{18} \mathrm{~m}^{-2}$ for NBA molecule $[14,15], \mu_{D}$ is the dipole moment of the individual molecule, $\varepsilon_{0}$ is the vacuum permittivity and $\varepsilon$ is the dielectric constant of SAM molecules, $(\varepsilon=5.3)$ [14]. Dipole moments of both molecules are calculated using Gaussian program and they are also verified in the literature [16]. Thus, the built-in potential of SAM based devices as /SAM/6T/Al may be written as follows:

$$
V_{b i}=\frac{1}{e}\left(\left(\phi_{\text {ITO }}-\phi_{\text {Al }}\right)+\Delta \phi\right)
$$

Note that the built in potential $V_{b i}$ can be reduced or enhanced depending on the sign of $\mu_{D}$ i.e. on the orientation of the dipole moment.

Finally one can write the expression of the open circuit voltage as:

$$
V_{\text {oc }}=\frac{1}{e}\left(\left(\phi_{\text {ITO }}-\phi_{\text {Al }}\right)+\Delta \phi\right)-\frac{k T}{e} \ln \left(\frac{g_{a}}{g_{c}}\right)
$$

\section{Results and Discussion}

\subsection{Devices with SAM of Thiol}

\subsubsection{Optical Characterization}

The UV-Visible absorption spectra have been record to a Carry 500 scan/UV-VIS-NIR spectrophotometer. Figure 3(a), shows the optical absorbance at normal incidence of 


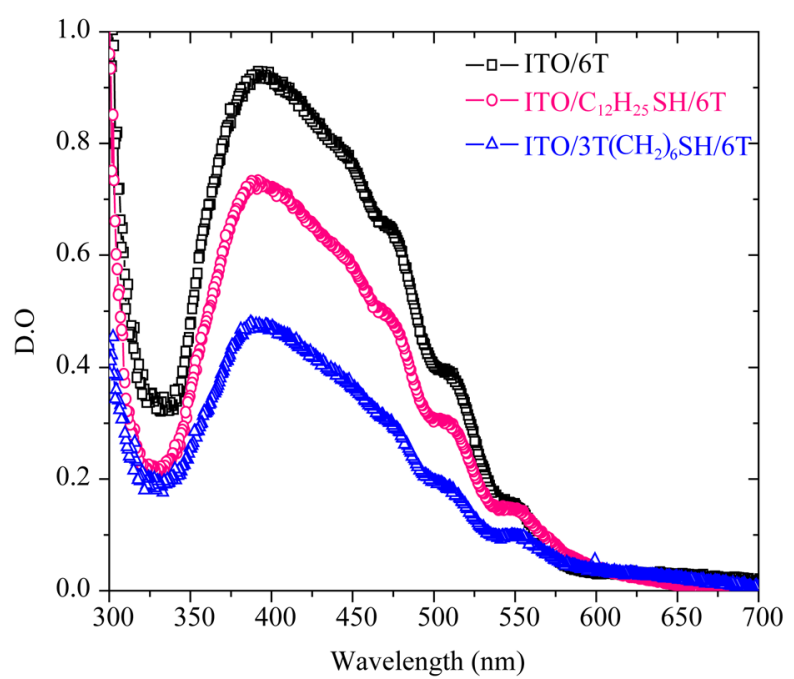

(a)

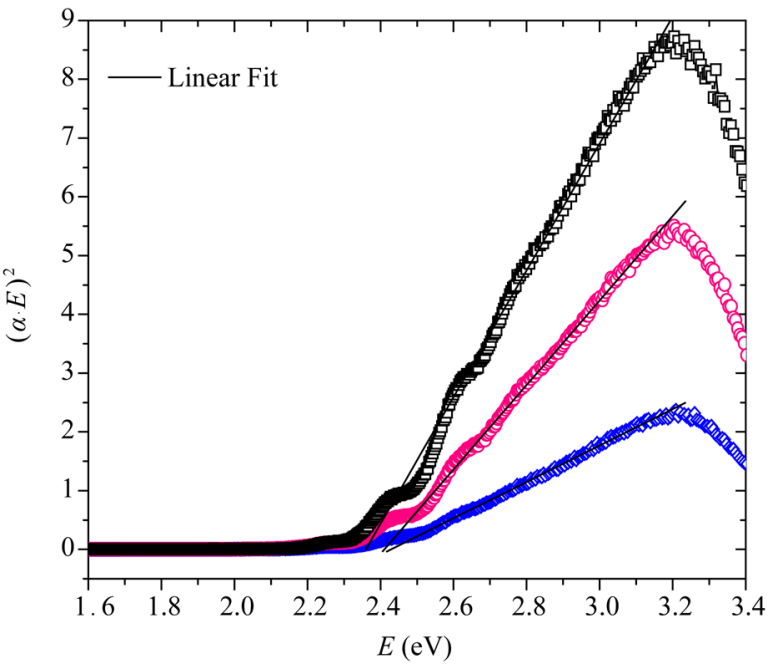

(b)

Figure 3. UV-visible absorption spectra of 6 T thin film deposited on SAM coated ITO and bare ITO substrates.

both samples: 6T on bare ITO and SAM coated ITO . Although the $6 \mathrm{~T}$ thin film layer is deposited simultaneously on both substrates, we notice that for the sample with SAM coated ITO the optical density (O.D.) is reduced by a factor 2 . This difference is not related to the film thickness bus may be accounted of the position of $\alpha$-6T molecules. In fact, electronic spectra of oligothiophene thin films are dramatically affected by the orientation of crystallites in the layer. Indeed, since the molecules of oligothiophene are lengthened and since the $\pi \pi{ }^{*}$ transition dipole moment $\boldsymbol{\mu}$ of these molecule is quasi-parallel to their long axis $\boldsymbol{L}$, then light absorption which is proportional to scalar product of the incident light electric field $\boldsymbol{E}$ by the transition dipole moment $\boldsymbol{\mu}$ is maximum when $\boldsymbol{E}$ and $\boldsymbol{\mu}$ are parallel and null when $\boldsymbol{E}$ and $\boldsymbol{\mu}$ are perpendicular [16]. Therefore the difference between the tree spectra may be accounted for by a better orientation of $6 \mathrm{~T}$ molecules in the case of $\mathrm{C}_{12} \mathrm{H}_{25} \mathrm{SH}$ and $3 \mathrm{~T}\left(\mathrm{CH}_{2}\right)_{6} \mathrm{SH}$ than in the case of bare ITO . Indeed, $6 \mathrm{~T}$ thin film seems to be much more organized with the SAM of $3 \mathrm{~T}\left(\mathrm{CH}_{2}\right)_{6} \mathrm{SH}$ then with $\mathrm{C}_{12} \mathrm{H}_{25} \mathrm{SH}$ and bare ITO, which can induce a quasi-epitaxial growth on terthiophene groups at the surface.

From the absorption spectra we can thus deduce the values of the band gap energy $\left(E_{g}\right)$ for sample with and without SAM of thiol by using the Tauc model [17]. This model supposes a parabolic variation of edge of the absorption band with energy of the photons. The absorption coefficient $\alpha$ is then associated to the energy of the photons $E$ and to the gap $\left(E_{g}\right)$ by the following relation which allows to extrapolate the bandwidth.

$$
\alpha E=A\left(E-E_{g}\right)^{n}
$$

where $E=h v$, effective energy of photon, $A$ is constant and $n$ is an index connect to the nature of electronic transition. We note according to Figure 3(b) that electronic transitions which appear in the absorption spectra are controlled by the vibrationnels levels from the molecule 6T. In fact, the permit transition of first excited state, correspond to the transition ${ }^{1} \mathrm{a}_{\mathrm{g}} \rightarrow^{1} \mathrm{a}_{\mathrm{u}}$ and contained in the plan (LM), allows us to deduce gap energy and the other transitions correspond of transitions towards the vibrationnels levels of the molecule $6 \mathrm{~T}$. This enabled us to adjust our spectrum on the first absorption band. We notice from adjustments results that the values of gap for $n$ $=1 / 2$ are in the same order of magnitude and are in agreement with that are deferred in the literature [16]. Moreover the best linear curve in the area of edge of absorption band corresponds to an average value of gap of the order $2.2 \mathrm{eV}$, and all the found values show that the gap of sexithiophene layer is independent of the functional surface.

Consequently, grafting of aliphatic self assemblies monolayer of thiols or with thiophene head group on ITO, emerge on the level of the orientation and the organization of the layers. This results are confirmed with the results of measurement of contact angle and the surface energy [11] that prove the better orientation of $\mathrm{C}_{12} \mathrm{H}_{25} \mathrm{SH}$ molecules towards the surface of ITO and the layer is relatively dense, but they have tendency to disorganize with the time of adsorption. In addition $3 \mathrm{~T}\left(\mathrm{CH}_{2}\right)_{6} \mathrm{SH}$ molecules are better organized on the surface and form a dipolar layer with the interface which would involve an electrical improvement of contact ITO.

\subsubsection{Current-Voltage Characteristics}

Figure 4 shows the tendency observed of ITO/6T/Al (REF) and devices with self assembled monolayer of 


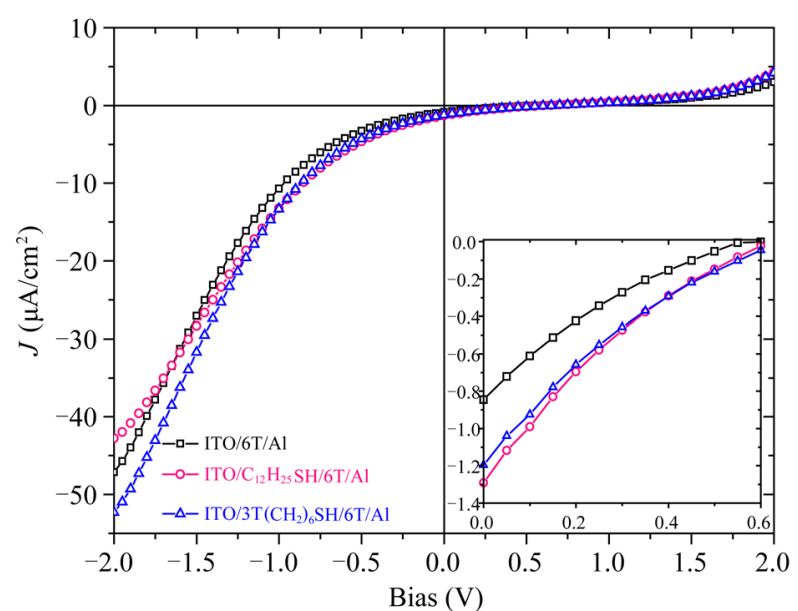

Figure 4. Current density characteristics at applied voltage illuminated with tungsten lamp for devices with SAMs of $\mathrm{C}_{12} \mathrm{H}_{25} \mathrm{SH}$ and (3T $\left.\left(\mathrm{CH}_{2}\right)_{6} \mathrm{SH} / 6 \mathrm{~T}\right)$, linear plot.

thiols under illumination by a tungsten lamp inside the Al. We clearly observe on the Figure $\mathbf{3}$ an effect of the light on I-V characteristics. It is a photocurrent that is strongly depends of the applied voltage. By observing photovoltaic parameters in Table 1, we remark clearly that the potential of open circuit and efficiency increased considerably for (ITO /SAM/6T/Al) compared to (ITO $/ 6 \mathrm{~T} / \mathrm{Al})$. Moreover, these results explain that SAM of thiol where the head groups are oligothiophenes present the best performances of the realized devices in spite of the fact that it absorbs a little luminous power that the two other devices. This would be show a better organization of the layer $[11,18,19]$ and induced a stronger mobility of the charges, affect the device efficiency. We could thus assure that the effect of the SAM containing thiols is limited to the photogeneration in volume and that really there is no direct interface effect on the dissociation of excitons. Thus, for devices with SAM of thiols the improvement of efficiency is probably imputable at the photogenerate free carrier of factors which are much less significant with tungsten lamp. In the continuation we will study from J-V characteristic under Xenon illumination, the effect of dipolar SAM molecules of benzoic acid in photovoltaic conversion.

\subsection{Devices with SAM of Benzoic Acid}

\subsubsection{Current-Voltage Characteristics}

The current-voltage characterization under illumination has shown different characteristics depending on the SAM nature (Figure 5, Figure 6). We notice that reverse biased photocurrent in NBA coated ITO samples is by far greater than the ABA coated ITO and the bare ITO samples, whereas its open circuit voltage is clearly lower than both of the other samples. On the other hand ITO/

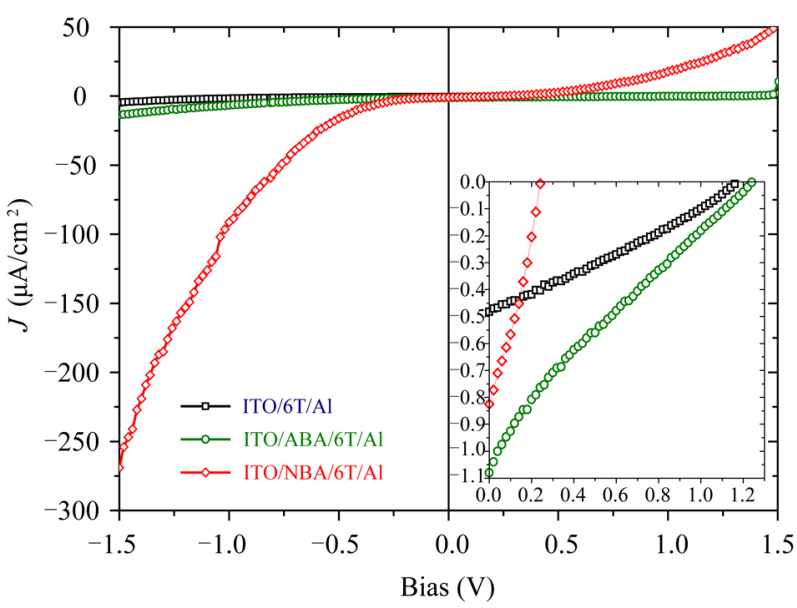

Figure 5. Current density characteristics at applied voltage illuminated with Xenon lamp for devices with SAMs of ABA and NBA, linear plot.

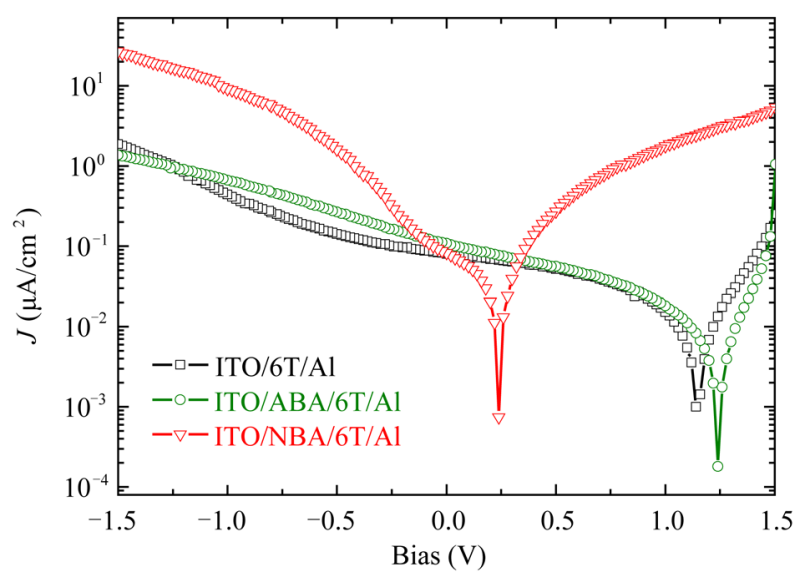

Figure 6. Current density characteristics at applied voltage illuminated with Xenon lamp for devices with SAMs of ABA and NBA, logarithmic plot.

ABA/6T/Al cell shows the largest short-circuit current $\left(J_{s c}\right)$ and also the largest open circuit voltage $\left(V_{o c}\right)$ (Table 2) summarizes the values of photocurrent densities $\left(J_{p h}\right)$, open circuit voltages $\left(V_{o c}\right)$ and the efficiencies with bare ITO and SAM-coated ITO samples.

It turns out that the conversion efficiency is dramatically affected by the grafting of the acid molecules on ITO. Indeed the efficiency of ITO/NBA/6T/Al is reduced nearly to the half of that of bare ITO sample, whereas in ITO/NBA/6T/Al sample the efficiency in almost twice that of bare ITO sample. The improvement of efficiency in the case of ABA can be accounted for by an enhancement of interfacial charge carriers photogénération due to the interaction of excitons with the dipole layer altogether with a suitable orientation of the dipole moment, that is the electric field lying in the dipolar SAM layer has the same orientation than that of the intrinsic electric 
Table 1. Open circuit voltage, current density and efficiency of conversion results of devices with SAM of thiols.

\begin{tabular}{ccccc}
\hline & $V_{o c}(\mathrm{~V})$ & $J_{c c}\left(\mu \mathrm{A} / \mathrm{cm}^{2}\right)$ & FF(\%) & $\eta \times 10^{-2}(\%)$ \\
\hline ITO/6T/Al & 0.55 & 0.85 & 18.5 & 2.4 \\
ITO/ ${ }_{12} \mathrm{H}_{25} \mathrm{SH} / 6 \mathrm{~T} / \mathrm{Al}$ & 0.60 & 1.29 & 18.7 & 4.0 \\
ITO/3T $\left(\mathrm{CH}_{2}\right)_{6} \mathrm{SH} / 6 \mathrm{~T} / \mathrm{Al}$ & 0,65 & 1.20 & 17.8 & 3.9 \\
\hline
\end{tabular}

Table 2. Open circuit voltage, current density and efficiency of conversion results of devices with SAM of benzoic acids.

\begin{tabular}{ccccc}
\hline & $V_{o c}(\mathrm{~V})$ & $J_{c c}\left(\mu \mathrm{A} / \mathrm{cm}^{2}\right)$ & FF(\%) & $\eta \times 10^{-2} \%$ \\
\hline ITO/6T/Al & 1.18 & 0.48 & 28.75 & 2.7 \\
ITO/ABA/6T/Al & 1.24 & 1.08 & 21.73 & 4.9 \\
ITO/NBA/6T/Al & 0.24 & 0.83 & 31.97 & 1.1 \\
\hline
\end{tabular}

field. Also, we can express this result by orientation of dipole moment of SAM in the direction favor of increase of interfacial energy between the ITO and the SAM layer. After collection of carriers at the interfaces we will have a significant generation of photocurrent.

\subsubsection{Orientation of the Dipole Moment on Photogeneration Carriers}

The change of ITO work function is related to the molecules adsorbed on the surface that are supporting a dipole moment owing to the presence of partial charges on the functional groups. These dipoles are aligned on the surface and form a dipolar layer which can be seen as an effective layer lying in a planar capacitor formed by charges of opposite sign at the edges of SAM layer and in which lays an intrinsic electric field (Figure 7). The orientation of the dipole either facilitates or inhibits the extraction of electrons or holes by the surface which entrains a shift of the photogenerated carriers resulting in a shift of potential surface [20-23].

We note that SAMs of benzoic acids increase or decrease the work function of the anode with the additional potential barrier with the interfaces depending on the orientation of the dipole moment of grafted acid molecules [24,25].

Therefore, at a first insight one can say that grafting SAMs with permanent dipolar moment molecules enhances the creation of carriers at the interface which may improve the performances of organic photovoltaic cells. But, the orientation of dipole layer will increase the built-in potential if the dipole layer field is in the same direction of the built-in electric field and vice-versa.

The experimental parameters according to Equation (11), (12) and (13) for bare ITO and SAM-coated ITO devices are summarized in (Table 3 ). We notice that ITO/NBA/6T/Al cells exhibit the largest photocurrent but the lowest open circuit voltage, whereas for ITO/
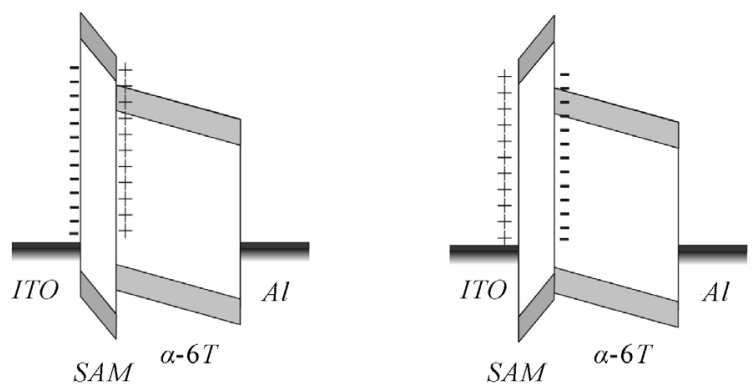

Figure 7. Photogeneration at interfaces.

ABA/6T/Al the $J_{p h}$ and $V_{o c}$ are slightly enhanced relatively to ITO/6T/Al. The NBA sample which has higher dipole moment than $\mathrm{ABA}$ is more efficient in creating photocarriers, but on the other hand it has a quite low open circuit voltage. The first feature can be interpreted by the strength of the dipolar moment which induces a quite strong field nearby the dipolar surface (Table 3); hence the dissociation rate of interacting excitons is considerably enhanced. Whereas the second feature is mainly due to the orientation of the dipolar moment, which results in an effective field oriented in the opposite direction of the built-in potential of the bulk sexithiophene thin film (Figure 7(a)). Thus the orientation of the dipolar moment of the SAM is a crucial factor in determining the open circuit voltage of a thin film photovoltaic cell. In fact, for ABA based samples in which the dipolar moment of the molecules is oriented such as the built-in potential is enhanced (Figure 7(b)), the open- circuit voltage as well as photocurrent are slightly enhanced compared to ITO/6T/Al samples.

In the NBA sample which has higher dipole moment than ABA is more efficient in creating photogenerated carriers, but on the other hand it has a quite low open circuit voltage. For NBA based samples, a remarkable decrease of built in potential that follow the magnitude 
Table 3. Results of photocurrent density, Open circuit voltage, build in potential and free-standing dipole moment of SAM of the devices.

\begin{tabular}{ccccccc}
\hline & Dipole moment (D) $[13]$ & $\Delta \phi(\mathrm{eV})$ & $J_{P h}\left(\mu \mathrm{A} / \mathrm{cm}^{2}\right)(@-1 \mathrm{~V})$ & $V_{O C}(\mathrm{~V})$ & $V_{b \mathrm{i}}(\mathrm{eV})$ & $g_{a} / g_{c}$ \\
\hline Bare ITO & - & - & 0.81 & 1.18 & 0.60 & $8.40 \cdot 10^{-11}$ \\
ABA & +2.64 & +0.38 & 3.31 & 1.24 & 0.98 & $2.55 \cdot 10^{-5}$ \\
NBA & -5.94 & -0.55 & 45.44 & 0.24 & 0.05 & $5.17 \cdot 10^{-4}$ \\
\hline
\end{tabular}

and the orientation of dipole moment. We can assume that strength of the dipolar moment induces a quite strong field nearby the dipolar surface.

\section{Conclusions}

The grafting of self assembled monolayers with thiols molecules and dipolar molecules of benzoic acid on ITO may be a fashionable way to improve photovoltaic performance of organic cells. Analyze of UV-Visible absorption spectra shows an effect of thiols SAM on the orientation of the sexithiophene molecule on the substrate (gap $2.2 \mathrm{eV}$ ), what can be related to the degree of organization of the thin layer that is better with the molecules $3 \mathrm{~T}\left(\mathrm{CH}_{2}\right)_{6} \mathrm{SH}$. The current vs. applied voltage characterisation show an enhancement of device efficiency that confirm the effect of thiols molecules on the photogeneration of free carriers in the bulk i.e. far from electrodes and contribute to photocurrent, due to the conjunction between the low mobility of free carriers in organic materials and their short lifetime. Moreover, it may be worth remembering that dipolar benzoic acids derivative (ABA and NBA) increase the efficiency of photovoltaic cells. This increase is significant especially for oriented dipole molecule of ABA at ITO/6T interface $(0.05 \%)$ reported with NBA. This improvement is affected by interaction of tightly bound Frenkel excitons with a surface dipole that may lead to efficient dissociation of geminate electron-hole pairs that has a significant effect on the photocurrent rate. However the contribution of photogenerated carriers to photocurrent is strongly dependent on dipole orientation. In fact the NBA compound has a large dipole moment but is oriented in the sight of reduction in photocurrent contrary to ABA. Then grafting strong dipole moment molecules oriented towards the cathode would provide a significant improvement of organic photovoltaic cells.

\section{Acknowledgements}

The author will like to thanks and express her gratitude to Dr. Fayçal Kouki and Prof. Habib Bouchriha, directors of research in UMAO (University El-Manar, Tunis) for their helpful and critical discussions to accomplish the study. I express also my thanks to Mr. Gill Horowitz and Mr. Philippe Lang, directors of research in ITODYS
(University Paris7) for their assistance and support in experimental studies.

\section{REFERENCES}

[1] G. Yu and A. J. Heeger, "Polymer Photovoltaic Cells: Enhanced Efficiencies via a Network of Internal DonorAcceptor Heterojunctions,” Science, Vol. 270, No. 5243, 1995, pp. 1789-1791. doi:10.1126/science.270.5243.1789

[2] P. Peumans and S. R. Forrest, "Very-High-Efficiency Double-Hetero-Structure Copper Pthalocyanine/ $\mathrm{C}_{60}$ Photovoltaic Cells,” Applied Physics Letters, Vol. 79, No. 1, 2001, pp.126-128. doi:10.1063/1.1384001

[3] G. Li and Y. Yang, "High Efficiency Solution Processable Polymer Photovoltaic Cells by Self-Organization of Polymer Blends,” Nature Materials, Vol. 4, No. 11, 2005, pp. 864-868. doi:10.1038/nmat1500

[4] C. L. Braun, "Electric Field Assisted Dissociation of Charge Transfer States as a Mechanism of Photocarrier Production," Journal of Chemical Physics, Vol. 80, No. 9, 1984, pp. 4157-4161. doi:10.1063/1.447243

[5] F. Kouki and H. Bouchriha, "Photogeneration Process in Pristine Sexithiophene Based Photovoltaic Cells," Organic Electronics, Vol. 11, No. 8, 2010, pp.1439-1444. doi:10.1016/j.orgel.2010.06.005

[6] S. F. Alvaradoand and D. D. C. Bradley, "Direct Determination of the Exciton Binding Energy of Conjugated Polymers Using a Scanning Tunneling Microscope,” Physical Review Letters, Vol. 81, 1998, pp. 1082-1085. doi:10.1103/PhysRevLett.81.1082

[7] X. Crispin, "Interface Dipole at Organic/Metal Interfaces and Organic Solar Cells," Solar Energy Mater and Solar Cell, Vol. 83, No. 2-3, 2004, pp. 147-168. doi:10.1016/j.solmat.2004.02.022

[8] D.H. Kim and S. Young Oh, "Effects of ITO Surface Modification Using Self-Assembly Molecules on the Characteristics of OLEDs," Ultramicroscopy, Vol. 108, No. 10, 2008, pp. 1233-1236. doi:10.1016/j.ultramic.2008.04.026

[9] H. Sirringhaus and R. Friend, "Integrated Optoelectronic Devices Based on Conjugated Polymers,” Science, Vol. 280, No. 5370, 1998, pp.1741-1744. doi:10.1126/science.280.5370.1741

[10] S. Goncalves-Conto and L. Zuppiroli, "Interface Morphology in Organic Light-Emitting Diodes,” Advanced Materials, Vol. 11, No. 2, 1999, pp.112-115. doi:10.1002/(SICI)1521-4095(199902)11:2<112::AID-A 


\section{DMA112>3.0.CO;2-Z}

[11] H. Bedis Ouerghemmi and F. Kouki, "Self-Assembled Monolayer Effect on the Characteristics of Organic Diodes," Synthetic Metals, Vol. 159, No. 7-8, 2009, pp. 551-555. doi:10.1016/j.synthmet.2008.11.031

[12] G. G. Malliaras and J. C. Scott, "Photovoltaic Measurement of The Built-in Potential in Organic Light Emitting Diodes and Photodiodes," Journal of Applied Physics, Vol. 84, No. 3, 1998, pp. 1583-1587. doi:10.1063/1.368227

[13] S. G. Ray and H. Waldeck, “Organization-Induced Charge Redistribution in Self-Assembled Organic Monolayers on Gold," Journal of Physical Chemistry B, Vol. 109, No. 29, July 2005, pp. 14064-14073. doi:10.1021/jp050398r

[14] H. Bässler and G. Vaubel, "Exciton-Induced Photocurrents in Molecular Crystals," Discussions of the Faraday Society, Vol. 51, 1971, pp. 48-53. doi: 10.1039/DF9715100048

[15] L. Zuppiroli1 and M. Grätzel, "Self-assembled Monolayers as Interfaces for Organic Opto-Electronic Devices," European Physical Journal B, Vol. 11, No. 3, 1999, pp. 505-512. doi:10.1007/s100510050962

[16] F. Kouki and F. Garnier, "Experimental Determination of Excitonic Levels in Alpha-Oligothiophenes,” Journal of Chemical Physics, Vol. 113, No. 1, 2000, pp. 385-391. doi:10.1063/1.481804

[17] J. Tauc and A. Vancu, "Optical Properties and Electronic Structure of Amorphous Germanium," Physica Status Solidi, Vol.15, No. 2, 1966, pp. 627-637. doi:10.1002/pssb.19660150224

[18] N. Camaioni and Giovanna Barbarella, "Branched ThioPhene-Based Oligomers as Electron Acceptors for Or- ganic Photovoltaics," Journal of Materials Chemistry, Vol. 15, No. 22, 2005, pp. 2220-2225.

doi: 10.1039/B503062E

[19] H. Hoppe and N. Serdar Sariciftci, "Polymer Solar Cells," Advances in Polymer Science, Vol. 214, 2007, pp.1-86. doi: 10.1007/12_2007_121

[20] J. W. King and S. P. Molnar, "Molecular Structural Index Control in Property-Directed Clustering and Correlation,” International Journal of Quantum Chemistry, Vol. 80, No. 6, 2000, pp. 1164-1171. doi:10.1002/1097-461X(2000)80:6<1164::AID-QUA3>3. $\underline{0 . \mathrm{CO} ; 2-\mathrm{C}}$

[21] I. H. Campbell and D. L. Smith, "Electrical Impedance Measurements of Polymer Light Emitting Diodes”, Applied Physics Letters, Vol. 66, No. 22, 1995, p. 3030. doi:10.1063/1.114267

[22] P. S. Davids and D. L. Smith, "Nondegenerate Continuum Model for Polymer Light - Emitting Diodes," Journal of Applied Physics, Vol. 78, No. 6, 1995, pp. 4244-4252. doi:10.1063/1.359886

[23] A. J. Cambell and D. G. Lidzey, "Space-Charge Limited Conduction with Traps in Poly (Phenylene Vinylene) Light Emitting Diodes,” Journal of Applied Physics, Vol. 82, No. 12, 1997, pp. 6326-6342. doi:10.1063/1.366523

[24] M. Carrara and L. Zuppiroli, "Carboxylic Acid Anchoring Groups for the Construction of Self-Assembled Monolayers on Organic Device Electrodes,” Synthetic Metals, Vol. 121, 2001, pp. 1633-1634. doi:10.1016/S0379-6779(00)00728-1

[25] H. Bedis, F. Kouki and H. Bouchriha, "Effect of Self Assembled Monolayers on Carrier Photogeneration in Organic Photovoltaic Cells," International conference ELECMOL'08 Grenoble-HMMNT-Minatec, 2008. 